\title{
La consolidación de la gobernanza empresarialista en la gestión urbana local. Un recorrido a través de las adaptaciones normativas e institucionales en la ciudad de Córdoba, Argentina
}

\author{
A consolidação da governança empresarial na gestão urbana \\ local. Um passeio pelas adaptações regulatórias e institucionais na \\ cidade de Córdoba, Argentina \\ The consolidation of entrepreneurial governance in local urban \\ management. A tour through the regulatory and institutional \\ adaptations in Córdoba city, Argentina
}

Natalí Peresini [a] [D

[a] Consejo Nacional de Investigaciones Científicas y Técnicas (CONICET). Córdoba, Argentina.

Cómo citar: Peresini, N. (2021). La consolidación de la gobernanza empresarialista en la gestión urbana local. Un recorrido a través de las adaptaciones normativas e institucionales en la ciudad de Córdoba, Argentina. urbe. Revista Brasileira de Gestão Urbana, v.13, e20200116. https://doi.org/10.1590/2175-3369.013.e20200116

\section{Resumen}

Desde los estudios urbanos críticos se señala que, desde hace más de tres décadas, se desarrolla un proceso de reconfiguración regulatoria e institucional para afianzar el proyecto neoliberal en las ciudades (Brenner et al., 2011; Peck et al., 2009). Parte fundamental de dicho proceso lo constituye la consolidación de la gobernanza empresarialista como modelo para la gestión urbana local, con el fin de asegurar la injerencia y la participación necesaria del sector privado para un orden urbano pro empresarial. Este artículo recoge los resultados parciales de una investigación en curso, atendiendo la evolución de las adaptaciones normativas e institucionales que se han incluido en la gestión urbana local en los últimos años. Para ello, mediante un enfoque cualitativo, se propone reconstruir la multiplicidad de normas, instrumentos y actores, así como también el nivel de institucionalidad que alcanzan, con el fin de caracterizar y describir la evolución histórica y coyuntural del marco regulatorio urbano en un caso de estudio. La genealogía construida permite evidenciar que en los últimos años se intensificó la incorporación de espacios e instrumentos de vinculación público-privada que permiten negociar las condiciones normativas en las cuales se desarrollan las operaciones, marcando un ciclo novedoso en la gestión urbana local.

Palabras clave: Gestión urbana local. Gobernanza urbana. Adaptaciones normativas e institucionales. Proceso de neoliberalización.

NP es arquitecta, miembro del equipo técnico del grupo Gestión integral del Hábitat en el Centro Experimental de la Vivienda Económica (CEVE), doctoranda en Estudios Urbanos en la Universidad Nacional de General Sarmiento y becaria doctoral CONICET, e-mail: natperesini@gmail.com 


\title{
Resumo
}

Estudos urbanos críticos indicam que, por mais de três décadas, um processo de reconfiguração regulatória e institucional está ocorrendo para fortalecer o projeto neoliberal nas cidades (Brenner et al., 2011; Peck et al., 2009). Uma parte fundamental desse processo é a consolidação da governança empresarial como modelo para a gestão urbana local, uma vez que garante a necessária interferência e participação do setor privado para uma ordem urbana orientada para os negócios. Este artigo coleta os resultados parciais de uma investigação em andamento, atendendo à evolução das adaptações regulatórias e institucionais que foram incluídas na gestão urbana local nos últimos anos. Para isso, propõe-se, por uma abordagem qualitativa, reconstruir a multiplicidade de normas, instrumentos e atores, bem como o nível de institucionalidade que eles alcançam, a fim de caracterizar e descrever a evolução histórica e conjuntural do marco regulatório urbano no caso de estudo. A genealogia elaborada mostra que nos últimos anos se intensificou a incorporação de espaços e instrumentos de parceria público-privada que permitem negociar as condições regulatórias em que as operações são realizadas, marcando um novo ciclo na gestão urbana local.

Palavras-chave: Gestão urbana local. Governança urbana. Adaptações regulatórias e institucionais. Processo de neoliberalização.

\begin{abstract}
Critical urban studies indicate that a process of regulatory and institutional reconfiguration has been underwey to strengthen the neoliberal project in cities for more than three decades (Brenner et al., 2011; Peck et al., 2009). A fundamental part of this is the consolidation of entrepreneurial governance as a model for local urban management. This process ensures the necessary interference and participation of the private sector for a business-oriented urban order. This article presents the partial results of reaserch. It shows to the evolution of regulatory and institutional adaptations that have been included in local urban management in recent years. Through a qualitative approach, it proposed to reconstruct the multiplicity of norms, instruments and actors, as well as the level of institutionality they reach, in order to characterize and describe the historical and conjunctural evolution of the urban regulatory framework in a study case. The constructed genealogy shows that in the last few years the incorporation of spaces and instruments of public-private linkage has intensified, allowing the negotiation of the normative conditions in which operations are developed, marking a novel cycle in local urban management.
\end{abstract}

Keywords: Local urban management. Urban governance. Regulatory and institutional adaptations. Neoliberalization process.

\section{El urbanismo neoliberal y la gobernanza urbana empresarialista como modelo de gestión local}

Los estudios urbanos críticos a lo largo de las últimas décadas, proponen analizar los vínculos entre los procesos urbanos y los económicos-políticos, los fenómenos provocados y problemáticas ligadas a ellos desde diversas escalas y dimensiones de análisis. Destacan que, en el escenario contemporáneo, se observa una profundización de los mecanismos que operan sobre la dimensión práctica del modelo neoliberal en el espacio urbano, lo que los autores han llamado "urbanismo neoliberal" (Harvey, 2007; Peck et al., 2009). Esta definición, apunta a exponer a las ciudades como espacios estratégicos para el avance de los proyectos de neo liberalización en sus múltiples dimensiones, señalando a las políticas urbanas y cada componente de la ciudad como objeto de negocio y especulación en el marco de una mercantilización expandida que conforma y sostiene lo que Harvey denominó "ciudades neoliberales" (2007). Peck y Tickell (2002) invierten la relación y proponen pensar en una "urbanización del neoliberalismo", considerando que se ha utilizado a la ciudad y su gestión como terreno de prueba de estrategias neo liberalizadoras. Ambas conceptualizaciones sostienen la importancia fundamental del espacio urbano en el desarrollo económico contemporáneo ya que su crecimiento y transformaciones son 
guiados principalmente por los intereses del mercado. Podría decirse entonces que el desarrollo del proceso de neo liberalización ha convertido a las ciudades en "laboratorios institucionales" para la experimentación de políticas neoliberales que provocan un cambio en la presencia, injerencia y responsabilidad de la gestión urbana.

Si bien es claro que los procesos mencionados son de alcance global y a nivel estructural, se entiende también que existen matices de acuerdo al contexto de aplicación. Esta aproximación nos refiere al concepto de "neoliberalismo realmente existente" (Brenner \& Theodore, 2002). Los autores proponen analizar los procesos de transformación urbana ligados al neoliberalismo, interpretándolos como complejas interfaces entre geografía, instituciones -con protagonismo del Estado- y sociedad, al interior de las cuales es posible identificar características específicas ligadas a los contextos nacionales, regionales y locales. Lo que se intenta destacar es la "inserción contextual" de los proyectos de reestructuración neoliberal que tienen al espacio urbano como protagonista y, junto con ello, prestar especial atención a los procesos locales para analizar las herencias de políticas públicas y prácticas regulatorias que se han desarrollado en el tiempo. En este sentido, se han producido numerosos antecedentes que trasladan estas contribuciones conceptuales anglosajonas al análisis de casos en diferentes locaciones y circunstancias socio-históricas (Díaz Orueta \& Lourés, 2013; Hidalgo \& Janoschka, 2014; Jiménez et al., 2018; Vicuña del Río, 2013).

En relación con lo anterior se reconoce simultáneamente la emergencia de nuevas tendencias en las prácticas de gestión urbana, las que Harvey (1989) definió y caracterizó como empresarialismo urbano ("urban entrepreneurialism"). Este modelo se focaliza en consolidar los procesos de mercantilización de la ciudad gracias a una toma de posición y un rol activo de la gestión local en la promoción de la actividad y el negocio urbano-inmobiliario como objetivo ineludible. Siguiendo al autor, la gobernanza urbana empresarialista implicó al menos tres grandes variaciones en torno a: la promoción de alianzas entre sector público y sector privado (a los fines de atraer fuentes de financiamiento, inversiones o fuentes de empleo); la lógica empresarial que orienta las decisiones; y, una marcada orientación a la economía política del lugar, en términos de alcance y escala de las intervenciones (López Morales et al., 2012).

Si nos enfocamos en el contexto regional, se distinguen una serie de cambios en las funciones de los gobiernos locales que se vinculan con los procesos antes mencionados. De acuerdo con la literatura especializada, se reconocen tres olas del municipalismo latinoamericano desde la década del noventa. La primera, con foco en lograr autonomía de las administraciones centrales y responsabilizándose de redes y servicios; la segunda, en los primeros años del siglo XXI, en torno al modelo de desarrollo urbano local y la competitividad interurbana; y, la tercera ola-contemporánea- caracterizada por la concertación entre el sector gubernamental, agentes empresariales y la participación ciudadana (Graglia, 2017). En resumen, para la gestión urbana local esta evolución implicó fundamentalmente sumar la obligación de fomentar y sostener el "desarrollo urbano" (DU), en tanto estrategia de competitividad, a la ya establecida responsabilidad en la prestación de servicios e infraestructura.

Los cambios registrados no pueden comprenderse sin vincular estas trasformaciones al proceso de globalización que se dio a partir de la década del noventa. Fenómeno que estuvo especialmente enfocado en las administraciones sub nacionales y que implicó un viraje en las competencias de los gobiernos locales. En Argentina este proceso se consolida a partir del cambio constitucional de 1994, que declara entre otras modificaciones, la autonomía municipal en el orden institucional, político, administrativo, económico y financiero. A partir de la descentralización impuesta, los gobiernos locales debieron reformular sus políticas urbanas con el fin de sostener dichas autonomías. En este escenario, gran parte de las decisiones de gestión urbana se orientaron a fomentar y alimentar las actividades relacionadas al complejo urbano inmobiliario como actor imprescindible, pasando de una dependencia de recursos ligadas al centralismo estatal a una búsqueda de recursos en los agentes empresariales. A tal efecto, se ejecutaron diversos mecanismos técnico-institucionales para la liberalización, desregulación y la 
facilitación de operaciones urbano-inmobiliarias como estrategia de captación de recursos y financiamiento para la gestión local.

\section{La reconfiguración regulatoria en la gestión urbana local}

En este punto, resulta fundamental relacionar el modelo de gobernanza urbana empresarialista a las sucesivas y sistemáticas adaptaciones regulatorias e institucionales en la gestión urbana local con el fin de facilitar operaciones urbano-inmobiliarias. La gobernanza es entendida como el modelo de gestión urbana que permite la aplicación articulada de nuevas modalidades de regulación surgidas en el transcurso del orden neoliberal (Araujo, 2010) y que, por sus características, logra consolidar la evolución de la gestión gubernamental hacia formas empresariales. Se destaca que lo realmente efectivo de dicho modelo es "una especie de mandato imperativo que vincula a los agentes políticos a las instrucciones de los grupos sociales dominantes" (Araujo, 2010, p. 8), presentado ante la sociedad como una discusión racional entre los grupos afectados y los representantes de gobierno mediante instancias de participación que legitiman las decisiones.

Desde los estudios urbanos críticos, se señala que la reconfiguración normativa e institucional que logra promover e instalar un Estado-empresario (Theodore et al., 2009) constituye una acción fundamental para lograr afianzar el proyecto neoliberal en este campo. Brenner et. al. (2011) proponen la noción de restructuración regulatoria para dar marco al patrón de cambios institucionales, regulatorios y socio-espaciales que se vienen sucediendo, ya que los procesos de mercantilización están siempre mediados por las instituciones estatales. Los autores la definen como un proceso a lo largo del tiempo en el cual es posible identificar el desmantelamiento de formas institucionales estatales que son ajenas a los principios neoliberales, coordinada con la promoción de nuevas modalidades de regulación y formas de gestión ligadas a la liberalización y desregulación para beneficiar los intereses del mercado.

Incorporar al análisis la evolución de las transformaciones institucionales y las experimentaciones regulatorias en torno a la ciudad en los últimos años, nos invita a pensar las expresiones contemporáneas como una instancia, una nueva fase, ya que sus formas y consecuencias continúan en desarrollo. En este sentido se advierte que las modalidades y alcances de la cooperación o coalición gubernamental con los agentes de mercado han evolucionado en el tiempo, incrementando su legalidad y legitimidad hasta conseguir la institucionalización de procedimientos y prácticas que le son favorables. A los fines de esta indagación, definimos la institucionalización como la capacidad monopólica del Estado desde la dimensión material, de legalizar y reglamentar prácticas a través de las instituciones que lo componen y desde la dimensión simbólica, al otorgarle legitimidad (Oszlak \& O’Donnell, 1982; Ouviña, 2002). En este sentido, la definición de la normativa urbana, sus indicadores, instrumentos y el nivel de institucionalidad que alcanzan, configura y describe la orientación del gobierno local, su interpretación en torno al rol que debe cumplir en las mediaciones con los actores privados y las referencias dominantes en las políticas urbanas. En relación con ello y como manifestación del avance del urbanismo neoliberal, se advierte que la gestión urbana basada en la gobernanza empresarialista ha logrado incorporar la cooperación o coalición gubernamental con los agentes de mercado en el plano formal, en los objetivos institucionales y en los marcos regulatorios.

El análisis propuesto en esta investigación dirige su atención a la configuración y las sucesivas adaptaciones que se han producido en el marco regulatorio, institucional y organizacional de la gestión urbana local, lo que supone reconstruir la multiplicidad de dispositivos, normas y actores que le han dado forma. Al mismo tiempo se intenta construir un análisis que dé cuenta de la variación histórica y coyuntural del marco regulatorio con el fin de problematizar y reconocer las condiciones, características y fases en favor del modelo de gobernanza empresarialista y el proceso de neo liberalización en la gestión urbana local. 


\section{Aspectos metodológicos de la investigación}

Este artículo refleja los avances producidos en el marco de un proyecto de investigación en curso ${ }^{1}$. Tanto en la indagación como en el desarrollo del presente texto se adoptó una estrategia metodológica de base cualitativa a través de un enfoque exploratorio-descriptivo con el fin de construir una genealogía, un recorrido por las adaptaciones normativas, organizacionales e institucionales que se han realizado en la gestión urbana local en favor de la incorporación y consolidación de prácticas vinculadas al modelo de gobernanza urbana empresarialista.

Como indican los autores de referencia el proceso de neoliberalización es multi-escalar y multidimensional, habilitando indagaciones amplias en todos los niveles estatales. En esta investigación se decidió enfocar el análisis en la gestión urbana local, por dos razones principales. La primera, ligada al marco legal-institucional que se aplica en Argentina en el cual los municipios son autónomos y jurídicamente responsables en lo que respecta a la legislación de las normas que regulan los procesos de creación y uso del suelo urbano (Levenzon, 2012). La segunda, en referencia al rol preponderante de las administraciones locales en el proceso de globalización y competitividad trasnacional desde la década del noventa. Esta decisión no implica dejar de lado la comprensión y caracterización del proceso en un contexto regional y global, a la vez que se impone leer sus efectos más allá del límite o jurisdicción política del caso que se propone en el análisis.

Como método de aproximación empírico se propone el estudio de caso, estrategia de investigación ampliamente utilizada en los estudios urbanos. El análisis se centra en la ciudad de Córdoba (Argentina), entre 1983 y 2019, procurando identificar y reconstruir la multiplicidad de dispositivos, normas, instrumentos y actores, así como también el nivel de institucionalidad que alcanzaron, con el fin de caracterizar y describir la evolución histórica y coyuntural del marco regulatorio e institucional de la gestión urbana en la ciudad. Para ello se elaboró una periodización ad hoc en la cual se identifican y caracterizan ciclos o fases de acuerdo a tres ejes: (1) la normativa urbana propuesta (explorando tipos de norma y objeto de regulación, modificaciones, adaptaciones); (2) la estructura orgánica institucional (incorporación de áreas específicas o actores encargados de intervenir y controlar la regulación y la gestión urbana); y (3), los mecanismos y modalidades de vinculación público-privadas (reconociendo actores y formas institucionales incorporadas y legitimadas).

En cuanto a la construcción y análisis de datos se optó por utilizar la técnica de triangulación (Benavides \& Gómez-Restrepo, 2005; Valles, 1999), ya que habilita el uso de fuentes y métodos tanto cuantitativos como cualitativos, como también el empleo de diversas estrategias para el análisis. En este sentido, se implementó la recopilación, cruce y análisis de fuentes secundarias como documentos públicos (ordenanzas, normativas y planes urbanos del gobierno local); de bibliografía específica; de documentos no oficiales (publicaciones en medios gráficos); y, la distinción y construcción del entramado de actores. Con estas fuentes y de manera articulada se procedió a analizar los datos obtenidos con el fin de definir características comunes que puedan señalar ciclos o fases verificando y comparando la información obtenida.

A continuación, se exponen los principales resultados del análisis.

\section{Un recorrido por las adaptaciones normativas e institucionales en la gestión urbana local (1983-2019)}

Creemos necesario iniciar el abordaje del caso de estudio haciendo un breve repaso por algunos indiciadores socio-económicos que nos ayudarán a entender la jerarquía, escala y funcionamiento de la ciudad de Córdoba. Como urbanización, es la segunda mayor poblada de la Argentina con una población de 1.329.604 habitantes, de acuerdo con los datos del último censo oficial (INDEC, 2010), aunque su

\footnotetext{
${ }^{1}$ Proyecto de investigación y tesis doctoral que se desarrolla en el programa de Estudios Urbanos de la Universidad Nacional de General Sarmiento (UNGS-Argentina) financiado por el Consejo Nacional de Investigaciones Científicas y Técnicas -CONICET-
} 
capacidad para retener habitantes ha ido en baja afectada principalmente por la movilización de la población hacia ciudades aledañas que integran su región conurbada. La misma conforma la segunda aglomeración del país en una ubicación central sumado al carácter de capital provincial que detenta, concentrando actividades de administración gubernamental, salud, educación, actividad productiva industrial y de servicios, además de un centro comercial consolidado desde hace más de cincuenta años. Los pilares de la economía cordobesa lo constituyen el sector de servicios, comercio e industria (destacándose el rubro automotriz). En esta línea, la mayoría de los ocupados del aglomerado Córdoba pertenece al sector servicios (58\%); seguido por el sector comercial con un 19\%; el empleo industrial con el 13\% y la construcción con un 10\% (Dirección de Estadísticas, 2017).

\section{El proceso de urbanización local}

Conforme datos oficiales, la ciudad cuenta con un ejido de $24 \mathrm{~km}$ por $24 \mathrm{~km}$ de lado, siendo el más extenso del país, equivalente a $576 \mathrm{~km}^{2}-57.600 \mathrm{~h}^{2}$ - y coincidente con los límites políticos del departamento Capital, lo que determina una densidad promedio de $2.308 \mathrm{hab} / \mathrm{km}^{2}$. Su trayectoria de urbanización comienza marcada por una estrategia de ocupación territorial común a las ciudades latinoamericanas colonizadas, una cuadrícula de base y un modelo de organización de acuerdo con las condiciones heredades. De dicha base se desprende la estructura física hasta la actualidad, conteniendo un centro histórico denso y consolidado, un anillo de barrios peri centrales conformados en una primera expansión durante el siglo XIX, un área intermedia de baja densidad compuesta por barrios residenciales originados durante el proceso de industrialización de la ciudad a mediados del siglo XX y una periferia con características disímiles que ha sido el foco de una serie de cambios recientes, tal como muestra la imagen 1.

1870

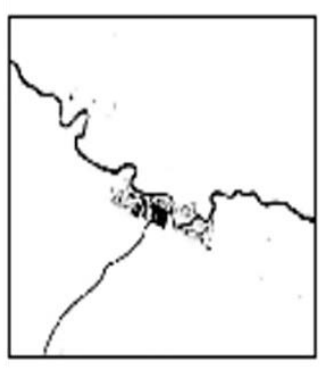

Población 36.223

1991

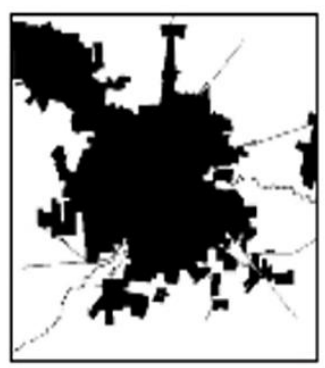

Población 1.179.372
1900

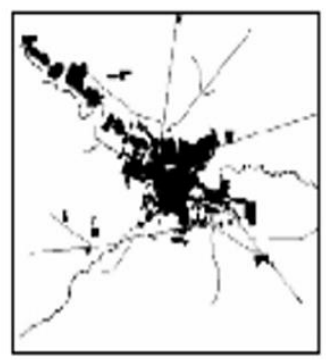

Población 72.500

2001

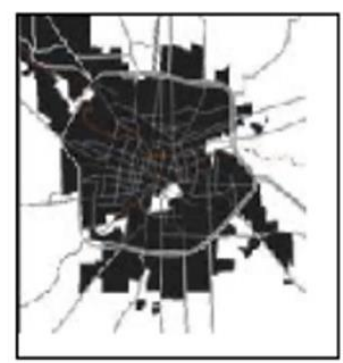

Población 1.284.582
1960

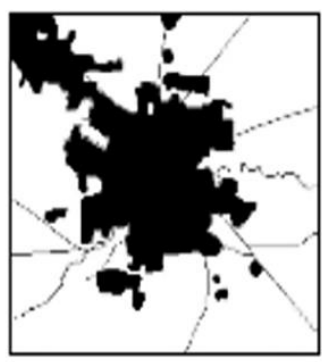

Población 509.163
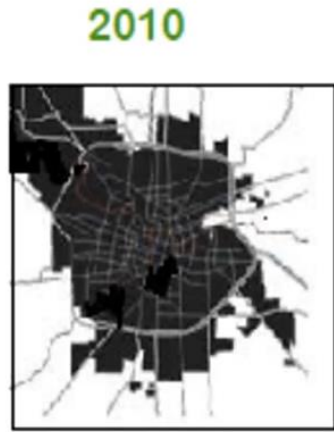

Población 1.329.604

Imagen 1 - Evolución del crecimiento de la mancha urbana de la ciudad de Córdoba y su población, por años censales. Fuente: Municipalidad de Córdoba. 
Desde la década del noventa se vienen señalando transformaciones cuantitativas y cualitativas que han influenciado la localización de la población, como muestra la distribución geográfica notablemente quebrada y dispar de la figura 1 y provocando fenómenos significativos en el proceso de urbanización.

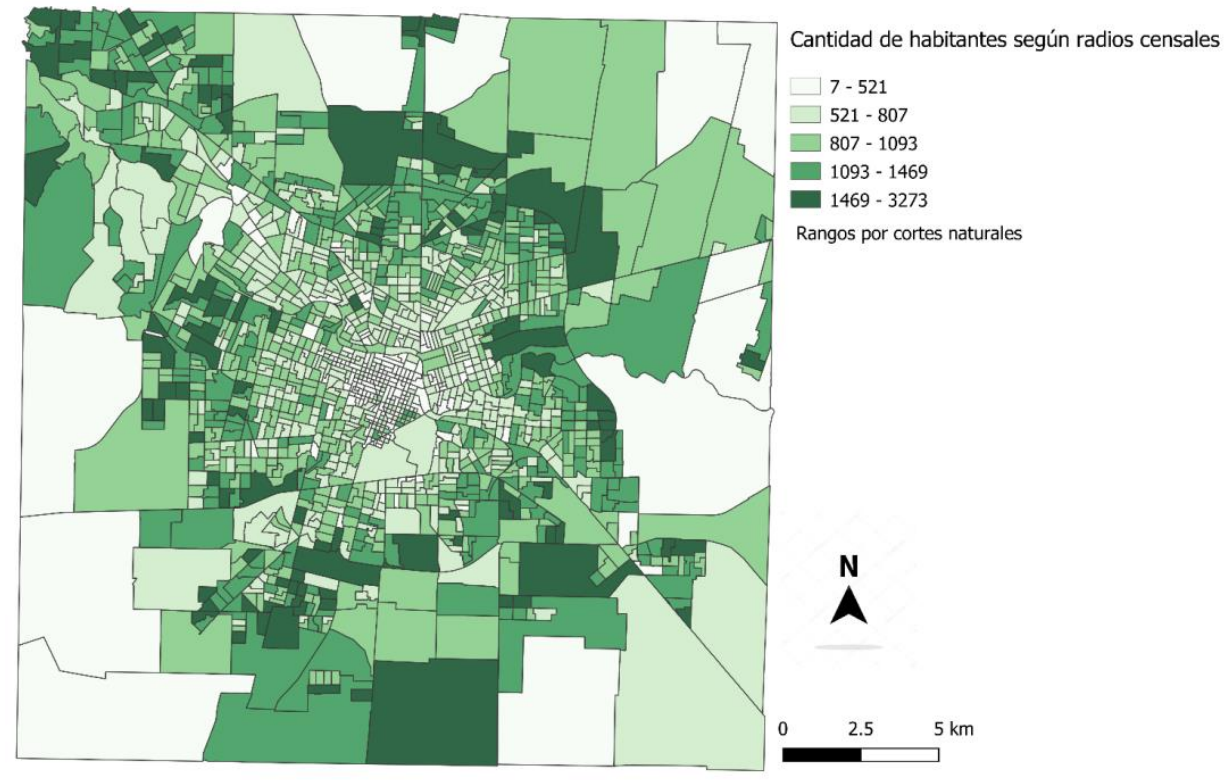

Figura 1 - Geo referenciación de población según densidad por radio censal. Ciudad de Córdoba, 2010. Fuente: elaboración propia en base a datos del INDEC. Censo nacional de población, hogares y viviendas, 2010. Datos procesados con Redatam+SP. Base geográfica disponible en http://www.indec.gob.ar/codgeo.asp

Expuestos los datos de indicadores socio-demográficos y la tendencia hacia la urbanización periférica y fragmentada nos debemos preguntar cuáles han sido las prioridades y orientaciones de la gestión urbana local y el marco político, institucional y regulatorio que ha avalado estos procesos.

\section{El primer marco normativo integral. La ciudad regulada (1983-1991)}

En los años ochenta, tras el regreso de la democracia como hecho determinante para el país, la gestión local asumió con fuerza la tarea de ordenar y reforzar las políticas públicas. Particularmente la gestión urbana intentó destacar la función de intervención y control estatal, de acuerdo también a la influencia de la agenda urbana internacional de retomar el poder tecnocrático del Estado y eficientizar la gestión pública (López, 2005; Van Lindert, 2016). En esta etapa se incorporó e institucionalizó el marco regulatorio urbano local, abocado a controlar las acciones privadas y normalizar bajo criterios estéticos y de higiene las condiciones de producción y ocupación de la ciudad. Para ello se siguieron los lineamientos predominantes en ese momento, tomando el modelo de zonificación para el mejor y más intensivo uso del suelo urbano, separando actividades residenciales, comerciales e industriales junto a la limitación de la extensión de la mancha urbana sobre áreas rurales. Bajo el Plan General de Desarrollo urbano (Iros, 1991) se propuso un cuerpo normativo basado en el control de tres áreas: el fraccionamiento (Ord. $\mathrm{N}^{\circ} 8060$ ); el uso (Ord. $\mathrm{N}^{\circ} 8133$ ) (imagen 2); y la ocupación del suelo (Ord. $\mathrm{N}^{\circ} 8256 / 1986$ para áreas intermedias y periféricas y la Ord. N 8057 para el área central). Mediante esta regulación el gobierno local intenta controlar las operaciones en diferentes escalas, tanto en lo referido al uso y disponibilidad de servicios del suelo como en las condiciones de fraccionamiento con el fin de generar patrones y condiciones "urbano-arquitectónicas" homogéneas. 


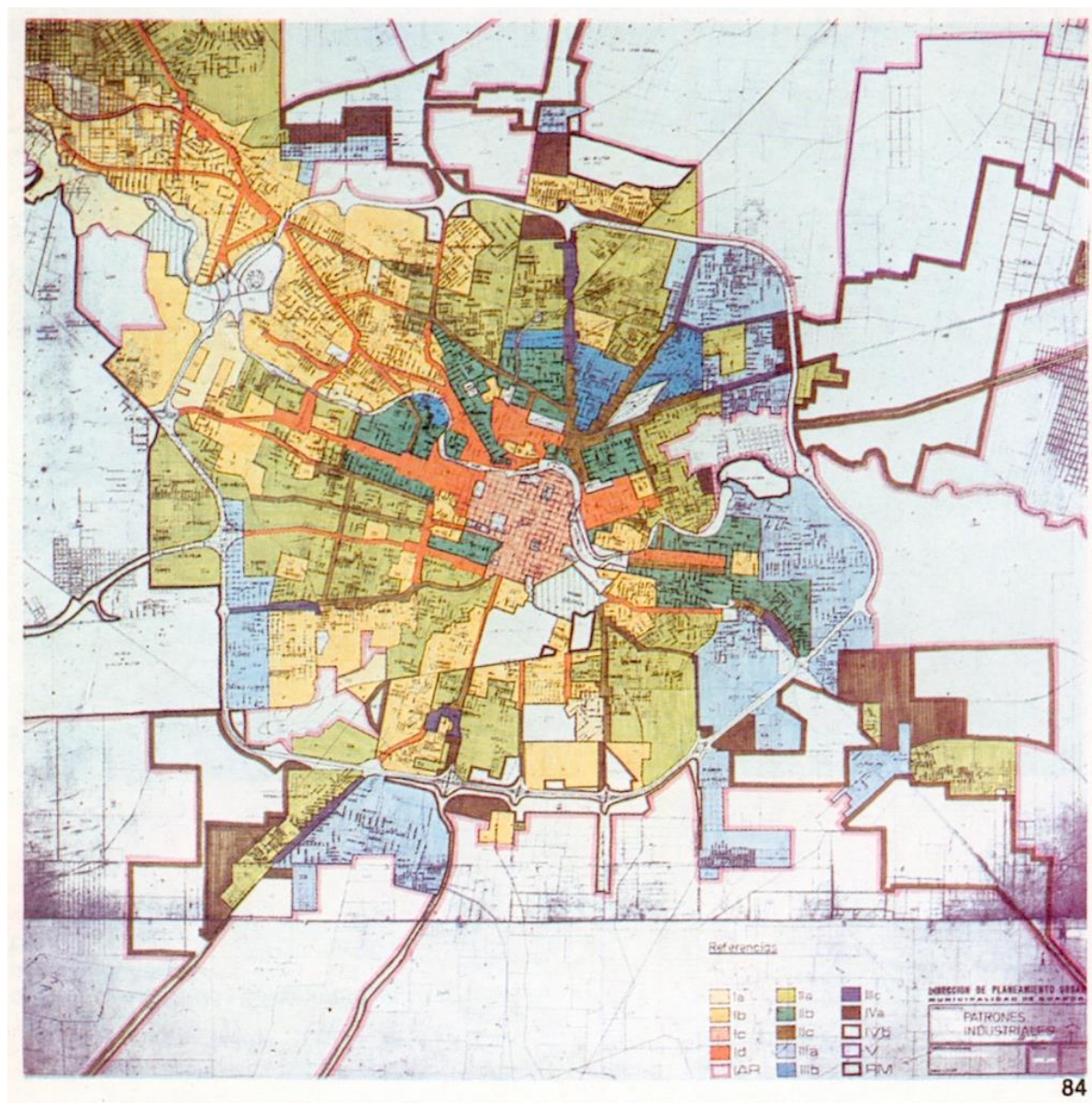

Imagen 2 - Espacialización de la ordenanza de usos del suelo №8133. Ciudad de Córdoba, 1985. Fuente: Archivo histórico municipal, Municipalidad de la ciudad de Córdoba.

La institucionalización de este conjunto de normas fue el principal y más duradero instrumento producido en estos años. El rol propuesto para el marco regulatorio refiere a una gestión urbana que establece limitaciones diferenciales sobre las propiedades de acuerdo con su localización, incide en los precios y provoca una distribución particular de la renta del suelo urbano. Un papel central en la dinámica económica de la producción urbana, al "constituirse como un instrumento capaz de configurar obligaciones y derechos" en torno a la propiedad y otorgar, mediante la asignación de índices, valorizaciones diferenciales (del Río et al., 2014).

Respecto de la estructura orgánica institucional, en este período se otorgó un lugar de mayor jerarquía y poder de decisión al área encargada de la gestión urbana, al ascender la dirección de planeamiento a Secretaría de desarrollo urbano. Tanto la elaboración como la configuración, en términos técnicos, del marco regulatorio fue realizada íntegramente por el cuerpo orgánico que integraba la Secretaría.

La planificación estratégica, la participación y la expansión de la ciudad (1991-1999)

A mediados de los años noventa se reemplazó el Plan General de Desarrollo Urbano por el Plan Estratégico Córdoba (PEC), cambiando el enfoque y las bases en los cuales se apoyaba la gestión urbana y marcando el inicio de un proceso con consecuencias hasta la actualidad. Los principios de la planificación estratégica, competitividad, productividad, gestión gerencial, apertura a las concertaciones público-privadas fueron los ejes de las trasformaciones durante estos años (Vanella et al., 2001). La necesidad de atraer inversiones para posicionar de manera competitiva a la ciudad en el orden global provocó la sucesión de una serie de adaptaciones en el marco regulatorio vigente. El mayor impacto en 
el orden normativo fue la decisión de no continuar con el control de la ocupación periférica sino todo lo opuesto, fomentarla a través del plan de descentralización municipal (construcción de centros de participación comunal) y el incentivo a la localización residencial en torno a estas "nuevas centralidades". Se avanza también sobre la flexibilización de requisitos en el parcelamiento y localización de barrios privados-cerrados, los usos permitidos en las zonas periféricas e intermedias, las condiciones de fraccionamiento y ocupación de esas mismas zonas, entre otras (Ord. №8606 en 1991 para urbanizaciones residenciales especiales; №9090, 9091, 9374, 9385, 9712, 9947, 9997, 10005, 10061, 10062 entre 1993 y 1999 para nueva zonificación; №9.395 para requisitos de edificación, №10.067 para usos, entre las más destacadas) (figura 2). Así, la función de control y regulación que se había asignado al cuadro de normas urbano-constructivas durante los años ochenta se revierte para armar una alianza estratégica con las inversiones privadas que permita el despliegue de los recursos económicos en la ciudad para su desarrollo, crecimiento y competitividad.

La estructura orgánica institucional se mantuvo relativamente estable en su conformación, con una configuración simple de Secretarías acorde a los servicios que prestaban los gobiernos locales durante estos años. Para 1996, se incorpora un nuevo espacio bajo la órbita de la Secretaría de gobierno, dedicado exclusivamente a llevar adelante el PEC: la Subsecretaria de planeamiento estratégico y desarrollo económico. La misma fue la encargada de gestionarlo bajo un claro objetivo económico, dejando la coordinación técnica de las obras planteadas a cargo de la Secretaría de desarrollo urbano, depreciando sus tareas y responsabilidades. Pareciera que, si bien en la estructura administrativa esta última conservaba el rango y la jerarquía, en este período fue reemplazada en la toma de decisiones y rol por este nuevo espacio que asume sus funciones de planificación de lo urbano con una clara orientación a la dimensión económica del territorio. Así, los principios gerenciales relacionados al paradigma de la planeación estratégica se convirtieron en los ejes de la gestión y la política urbana, conceptos como eficiencia, instrumentos, modernización aparecen en el entramado institucional y en el gobierno local para referirse al desarrollo urbano (Marianacci, 2000; Municipalidad de la Ciudad de Córdoba, 1996)

Bajo los objetivos del PEC, la promoción de un "nuevo relacionamiento público-privado" se posicionó como uno de los ejes centrales del plan junto a la necesidad de generar formas asociativas novedosas para la gestión urbana. Lo que se promovió concretamente, fueron modalidades de asociación no sólo respecto de las necesidades físico-espaciales (como pudieron ser algunos formatos emparentados a la realización de grandes obras de infraestructura), sino fundamentalmente centradas en el hecho urbano como un hecho económico. En este marco se desarrollaron concertaciones público-privadas en tanto acuerdos informales para la atracción de inversiones y la instalación en la ciudad de grandes firmas comerciales. Dichas concertaciones lograron operacionalizar excepciones particulares a grandes emprendimientos, como shoppings o centros comerciales, con el argumento de dinamizar las inversiones, la oferta inmobiliaria y conseguir la modernización de la ciudad siguiendo los preceptos globalizadores. La relevancia de estos casos como antecedentes radica en que la gestión urbana local acepta las decisiones y modificaciones normativas como parte de las reglas de juego con el fin de captar y atraer inversiones empresariales y alcanzar los objetivos del plan urbano. 


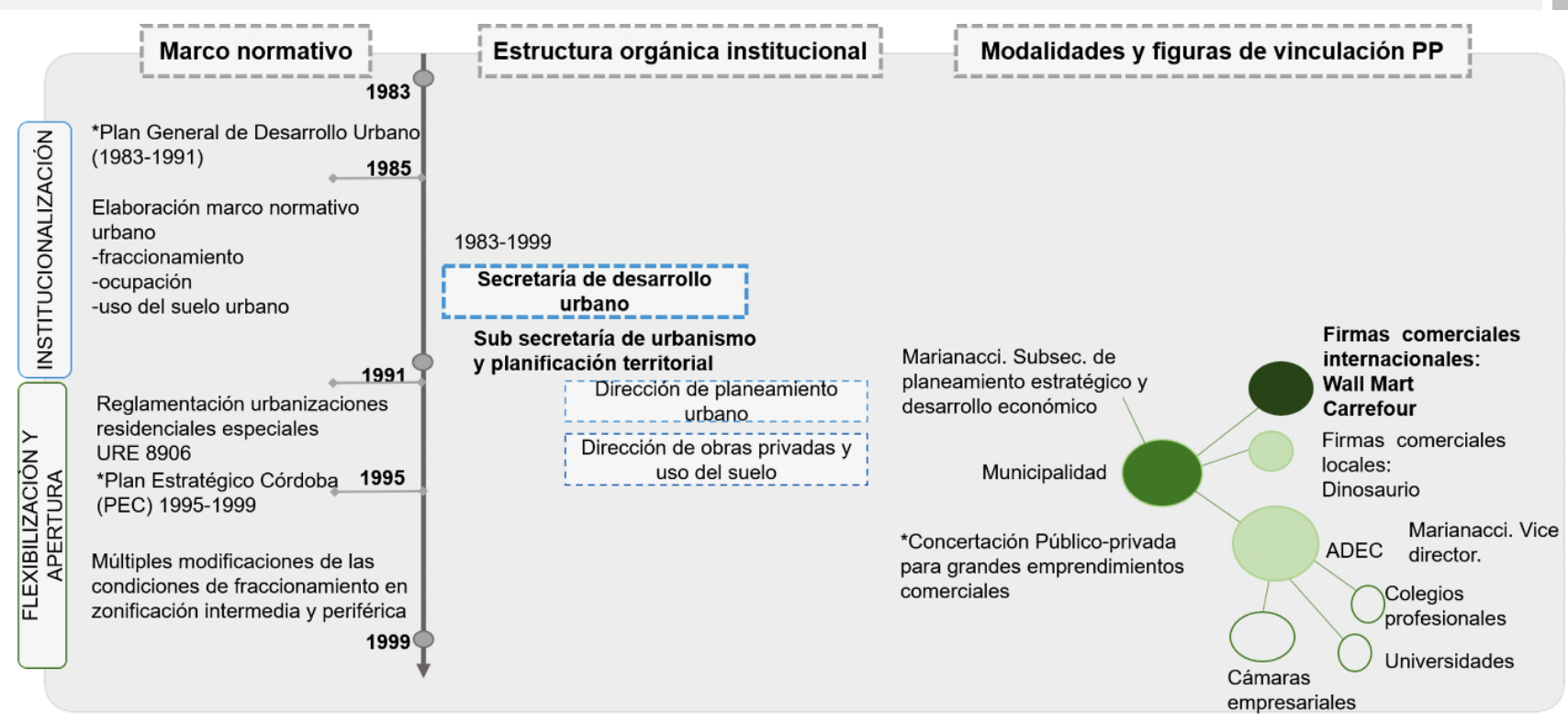

Figura 2 - Esquema síntesis de fases entre 1983-1999 según caracterización de normativa urbana propuesta, estructura orgánica institucional y mecanismos y modalidades de vinculación público-privadas. Ciudad de Córdoba. Fuente:

Elaboración propia de acuerdo con documentos oficiales.

Durante este período, surgió una primera oleada de políticas urbanas de corte neoliberal, caracterizadas no sólo por los cambios o excepciones al marco regulatorio urbano con el fin de incentivar inversiones privadas, sino también por abrir el espacio de decisión a los actores empresariales mediante el formato de concertación público-privado y la creación de nuevas institucionalidades mixtas. Éstas se organizaron por fuera de la estructura orgánica municipal, con el fin de cumplir la consigna de participación en las decisiones de políticas públicas, aunque incorporó casi exclusivamente a los actores empresariales. En coherencia con los objetivos de competitividad y desarrollo urbano-económico se conformaron y habilitaron la incubadora de empresas y la Agencia para el Desarrollo Económico de la Ciudad de Córdoba (ADEC). Al calor de estas nuevas instituciones, la idea de gestión público-privado tomó mucha fuerza bajo este plan y fue considerada como un indicador de éxito (ADEC, 1997). De estas "nuevas institucionalidades", la ADEC es la única que ha sobrevivido al paso de la propia gestión impulsora e incluso se ha fortalecido ocupando cada vez más espacios de decisión y consulta. Cabe destacar que estos espacios público-privados van a sufrir un corrimiento o debilitamiento de su rol y protagonismo en la primera década del 2000 retomando su lugar y participación cada vez mayor en la gestión entre 2011 y 2019.

Tanto la creación de "nuevas institucionalidades mixtas" como las operaciones de concertación fueron una innovación de este período que van a marcar el inicio de un ciclo en la gestión urbana local orientada a vincularse como un actor más en el mercado. Se atraviesa una transición de la planificación y gestión urbana con un sesgo físico-espacial a una condicionada por las variables económicas, permitiendo la participación de sectores industriales, comerciales y financieros en las decisiones respecto del territorio. La posición ganada del sector privado ya no va a retroceder, sino que se irá acrecentando en los años siguientes, institucionalizando su participación bajo distintos instrumentos y mecanismos y convirtiéndose en una voz de peso, legitimidad y con poder de habilitar o no la aprobación del proceso técnico-político de la planificación urbana durante la próxima década.

La gobernanza urbana empresarialista realmente existente: la gestión local y la presión de los actores del complejo urbano inmobiliario (1999-2011)

Posteriormente a la crisis económica-política-institucional que se dio en el país entre 2001 y 2003, los efectos de la devaluación sobre la moneda nacional y el aumento de rentas extraordinarias gracias al 
precio de las commodities agrícolas ${ }^{2}$ fueron muy positivos para el sector constructivo-inmobiliario, ya que se privilegió esta actividad como estrategia de capitalización de activos y reserva de valor. Estas variables implicaron también cambios en la escala y modelos de negocios en las principales empresas desarrollistas de la ciudad, incidiendo fuertemente en la gama de operaciones, en la movilización del negocio inmobiliario (Capdevielle, 2016; Cisterna et al., 2012) a la vez que produjeron saltos importantes en el valor del suelo urbano (Dávila, 2013; LaVoz, 2013). Este auge o "boom inmobiliario" (Baer, 2008) posicionó al sector como actor de peso e influencia en las decisiones de gestión urbana, poniendo en tensión las decisiones establecidas en el marco regulatorio urbano.

En paralelo, una operación de importancia y con efectos en los períodos siguientes fue la conformación de la Cámara de Empresarios Desarrollistas Urbanos de Córdoba -CEDUC- en 2004, en la cual los empresarios del sector se organizaron como gremio. En la presentación oficial declararon tener como objetivo "contribuir con el desarrollo urbano en la ciudad de Córdoba, establecer mecanismos de participación de las decisiones que involucren al sector, defender el cumplimiento del marco regulatorio y consolidar la seguridad jurídica" (LaVoz, 2004). En este escenario se puso en juego la capacidad de acción del sector empresarial y se da cuenta también de su interés en lograr incentivos específicos del sector público.

Durante este período se sucedieron múltiples intentos para consensuar un plan urbano, todos ellos sin éxito, manifestando una paulatina separación de la planificación urbana como actividad tradicionalmente vinculada a la gestión de la ciudad. En su reemplazo, se dio cada vez más lugar a la implementación de instrumentos "operativos y flexibles" con alcance sectorial y finalidades específicas, en mayor parte vinculados a la recuperación del plusvalor del suelo urbano y para la dinamización de la actividad constructiva-inmobiliaria.

Respecto a lo normativo, en este ciclo no sólo se van a flexibilizar los límites de las áreas dispuestas a la urbanización y sus condiciones como en el período anterior, sino que hacia el final del periodo van a surgir nuevos mecanismos para la negociación de las normas; un cambio de orientación que avanza de la flexibilización a la mercantilización. La evolución de las adaptaciones normativas demuestra que la regulación no sólo cumple un rol como requisito legal y burocrático para la producción urbanoinmobiliaria, sino que se convierte en el campo de juego donde se demuestra los alcances e influencia de cada sector.

En este período se identifican tres etapas que responden a cada gestión municipal: la primera, signada por la flexibilización de la normativa vigente sobre las zonas y áreas establecidas en el período anterior; la segunda, cargada de tensiones, marchas y contramarchas relacionadas a la presión del complejo urbano inmobiliario respecto a la habilitación de nuevas áreas y oportunidades de inversión; y, la tercera vinculada directamente a la apertura de las negociaciones y la mercantilización del marco regulatorio urbano dando inicio a un nuevo momento en la relación gestión urbana-empresarios. Respecto de esta última, en 2007 comienza un ciclo de institucionalización de instrumentos y espacios de participación empresarial que va a exponer el poder de injerencia del sector ligado al desarrollo urbano, una expresión de la gobernanza urbana empresarialista realmente existente. La mayor novedad en este ciclo lo va a constituir la habilitación e implementación de instrumentos centrados en la recuperación de plusvalor, nos referimos a la ordenanza de Obras Complementarias (Ord. №11.272, 2007) y la firma del primer Convenio Urbanístico (Ord. №11.545, 2008). Ambos comparten el objetivo de negociar las condiciones normativas en las cuales se desarrollan las operaciones inmobiliarias, modificaciones que otorgan beneficios sobre índices constructivos o usos del suelo que potencian los rendimientos económicos de los emprendimientos, a cambio de dejar un porcentaje del plusvalor obtenido para el municipio.

Así también, las repetidas variaciones de la representación partidaria de la gestión municipal y con ello en el enfoque en la política urbana fue un signo de estos años. Este último punto fue determinante

\footnotetext{
2 Se habla de commodities para referirse a materias primas o bienes primarios con bajo nivel de especialización e industrialización que se logran comercializar en los mercados internacionales a iguales valores en dólares.
} 
para los cambios en la estructura orgánica institucional durante este periodo, adaptaciones que se concentraron en contener y responder la demanda de los actores empresariales ligados a la actividad urbano-inmobiliaria local. Se produjeron constantes modificaciones en la planta de funcionarios y con ello en el enfoque de la gestión urbana y sus vínculos con el sector empresarial.

Estas variaciones transparentan a los agentes de la gestión urbana como una línea de poder estrechamente ligada a los actores más influyentes del desarrollo urbano a la vez que revelan una fuerte tensión e interés sobre las decisiones en torno a la regulación. Incluso es a partir de la demanda empresarial que se generaron espacios de consultoría, al interior de la estructura orgánica municipal. Ejemplo de ello fue el consejo de planificación urbanística -CPU-, una experiencia similar a la del PEC de los años noventa, aunque esta vez conformada como un espacio inter actoral al interior de la estructura municipal para representar la concertación público-privada. El espacio fue propuesto como consultivo a los fines de fomentar la participación y articulación con otros sectores y actores sociales con el fin de debatir las propuestas sobre modificaciones al marco regulatorio urbano, iniciativas de los empresarios y otras demandas de los actores involucrados en la construcción de la ciudad, de acuerdo con la concepción de la gestión municipal (Guevara, 2008). Concebido bajo la consigna de generar líneas de acción para un plan director urbano, el CPU logra en cambio proponer una serie de instrumentos de gestión que suponen una relación más directa entre las necesidades del sector privado y las posibilidades de "facilitar e impulsar el desarrollo urbano" del municipio. El espacio resultó funcionar como una excelente plataforma que posibilitó y legitimó el uso e implementación de los instrumentos; exitoso a los ojos de los desarrollistas, ya que lograron "ingresar" a la mesa de las decisiones, acercar sus intereses particulares y legitimar sus necesidades. En términos de la gobernanza urbana, lograron operacionalizar un cogobierno respecto de la gestión urbana (figura 3).

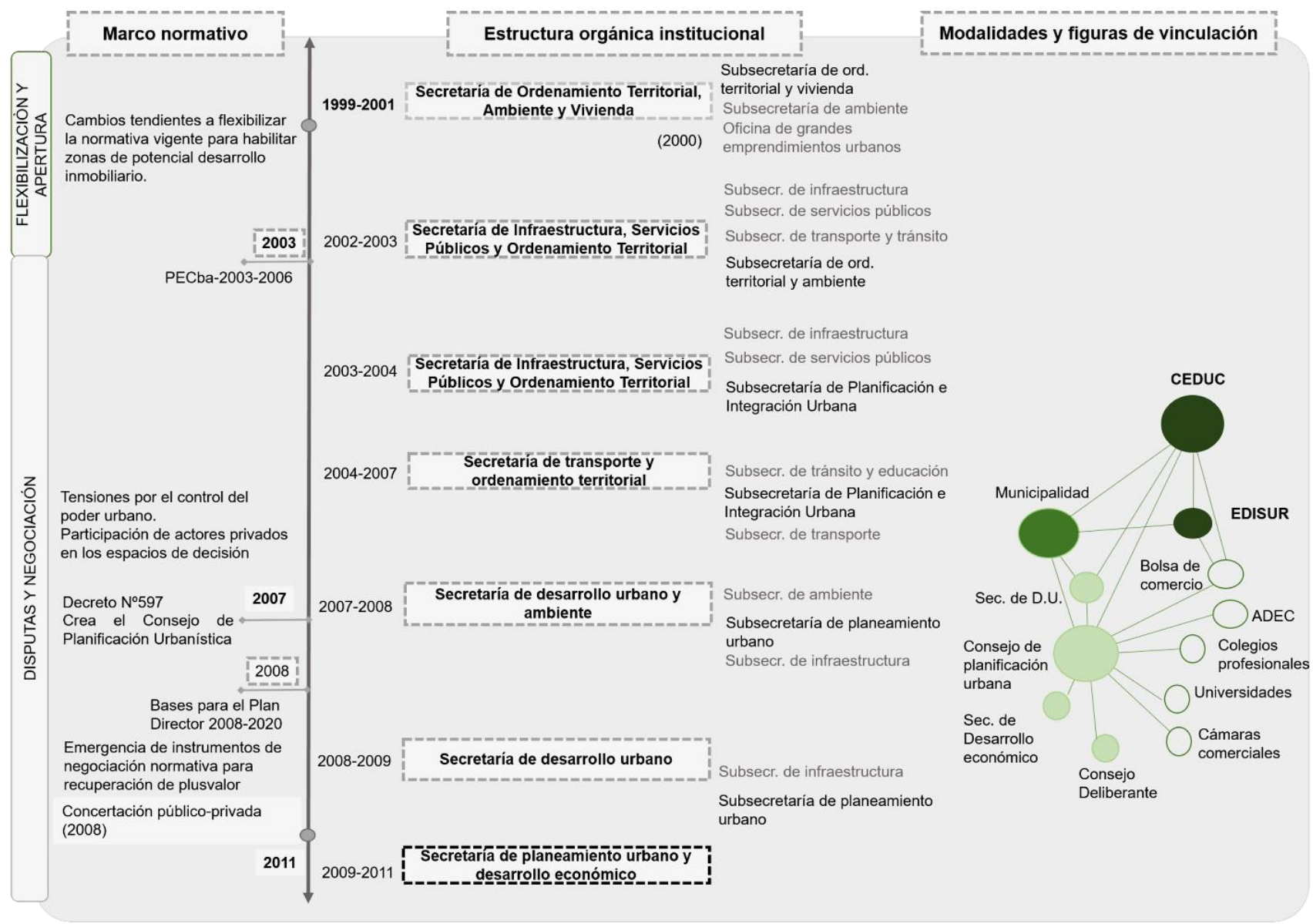

Figura 3 - Esquema síntesis de fases entre 1999-2011 según caracterización de normativa urbana propuesta, estructura orgánica institucional y mecanismos y modalidades de vinculación público-privadas. Ciudad de Córdoba. Fuente: 
En suma, la clave de este ciclo fue asumir la capacidad de injerencia del sector empresarial en las decisiones y con ello abrir la Secretaría de desarrollo urbano a la recepción de pedidos y exigencias de los empresarios relacionados a la actividad inmobiliaria, dando inicio a un nuevo momento en la relación gestión urbana-empresarios. A partir de ello, se registraron innovaciones instrumentales que van a alterar no sólo la función e injerencia del marco regulatorio urbano vigente sobre el "desarrollo urbano" sino la impronta y la lógica de la gestión urbana posterior.

\section{La mercantilización del marco regulatorio urbano como eje de la política y la gestión urbana (2011-2019)}

En el último ciclo de esta periodización, 2011-2019, las innovaciones sobre la normativa vigente fueron escasas, apelando a la gran efectividad que tuvieron los instrumentos implementados desde 2007 (figura 4). Los mecanismos incorporados fueron determinantes en materia normativa, dando cuenta de una evolución en el rol del marco regulatorio, la orientación de la gestión y las políticas urbanas partiendo de un paradigma regulador iniciado en la década del ochenta a uno negociador. En líneas generales, cada una de las instancias de flexibilización y apertura para los desarrollistas inmobiliarios descriptas anteriormente, marcaron un avance progresivo hacia la mercantilización de la normativa urbana que va a encontrar en la figura de Convenios Urbanísticos su mayor y mejor expresión. Es fundamental para comprender los eventos de este ciclo la injerencia de la CEDUC que, si bien comenzó en 2004, hacia finales de la década pasada logró influir de manera concreta sobre las decisiones en materia de gestión urbana, así como también su poder para legitimar o no las decisiones institucionales referidas particularmente al marco regulatorio urbano.

El período muestra que la flexibilización de la normativa como mecanismo de adecuación a los negocios inmobiliarios ya resultaba insuficiente. Sumado a ello, la concentración de operaciones y poder de los actores desarrollistas logró consolidarse y generó nuevas demandas respecto de las condiciones en las que pretendían desarrollarse. En esta última etapa y bajo estos mecanismos, la participación de los empresarios desarrollistas ya no se dio vía consulta o participación en la configuración de planes y programas sino directamente en la negociación de las condiciones. Como resultado, se institucionaliza la figura de Convenios Urbanísticos (Ord. №12077, 2012) normalizando su implementación como instrumento específico para la negociación regulatoria a cambio de un porcentaje del plusvalor generado por los beneficios dispuestos.

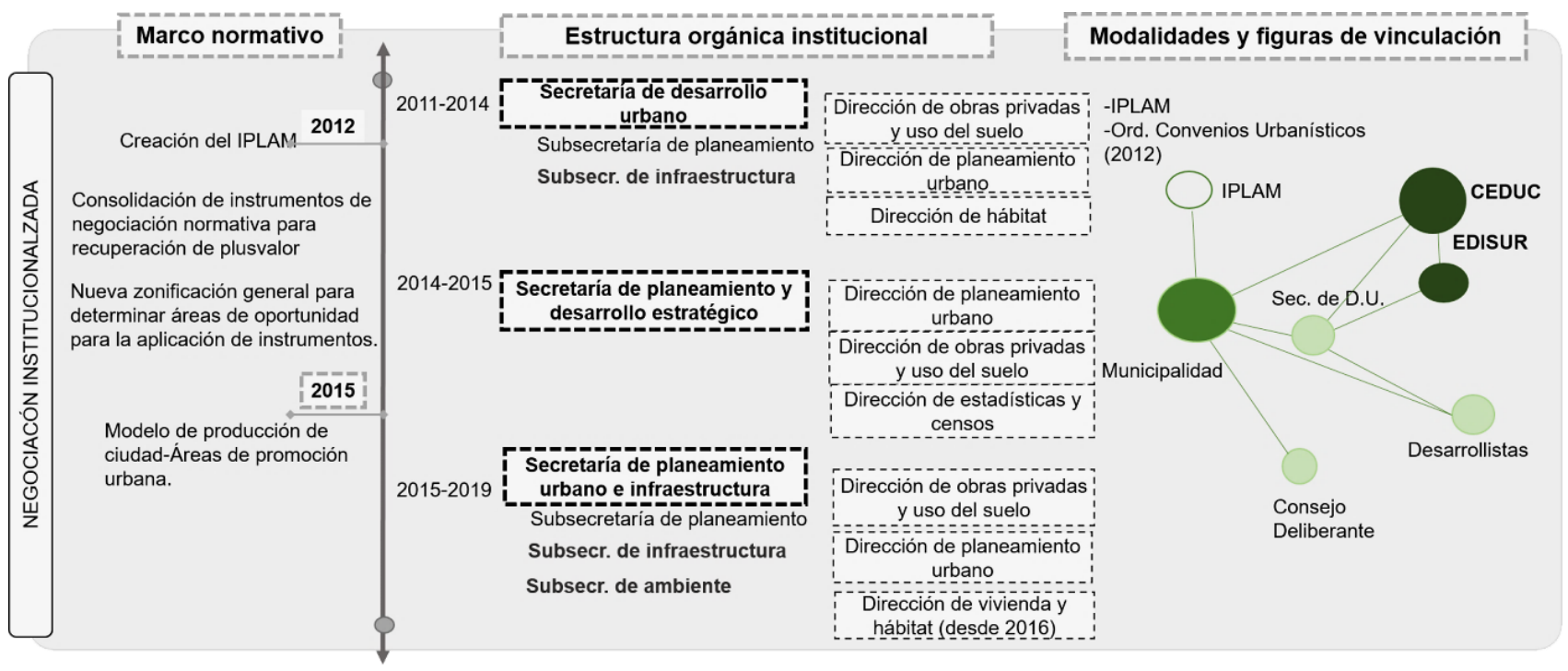

Figura 4 - Esquema síntesis de fase entre 2011-2019 según caracterización de normativa urbana propuesta, estructura orgánica institucional y mecanismos y modalidades de vinculación público-privadas. Ciudad de Córdoba. Fuente: 
En este recorrido temporal se hace evidente que las presiones del sector empresarial sobre las decisiones de políticas urbanas y sobre el marco regulatorio han sido exitosas, consiguiendo beneficios en favor de sus intereses particulares. En tanto logran la facilitación, flexibilización y la negociación de las condiciones en las cuales deben actuar, sin haber afectado o modificado en general las condiciones estructurales sobre las cuales se rige la producción urbana. En este contexto, desde la gestión municipal y su cuerpo orgánico se propició el uso y legitimización de instrumentos específicos, de aplicación particular y con una clara orientación financiera en pos de garantizar recursos para el municipio valiéndose de la recuperación de plusvalor de la renta del suelo urbano.

\section{Reflexiones de cierre}

En el relevamiento, sistematización y recorrido expuesto se evidencia la importancia de la gestión urbana local como espacio institucional y material en el cual se revela la evolución del proceso de neoliberalización, reconociendo las distintas instancias y adaptaciones regulatorias e institucionales a favor del mismo. A modo de síntesis en la figura 5 se representa de forma integral la periodización y caracterización elaborada de acuerdo con la indagación en el caso de estudio propuesto.

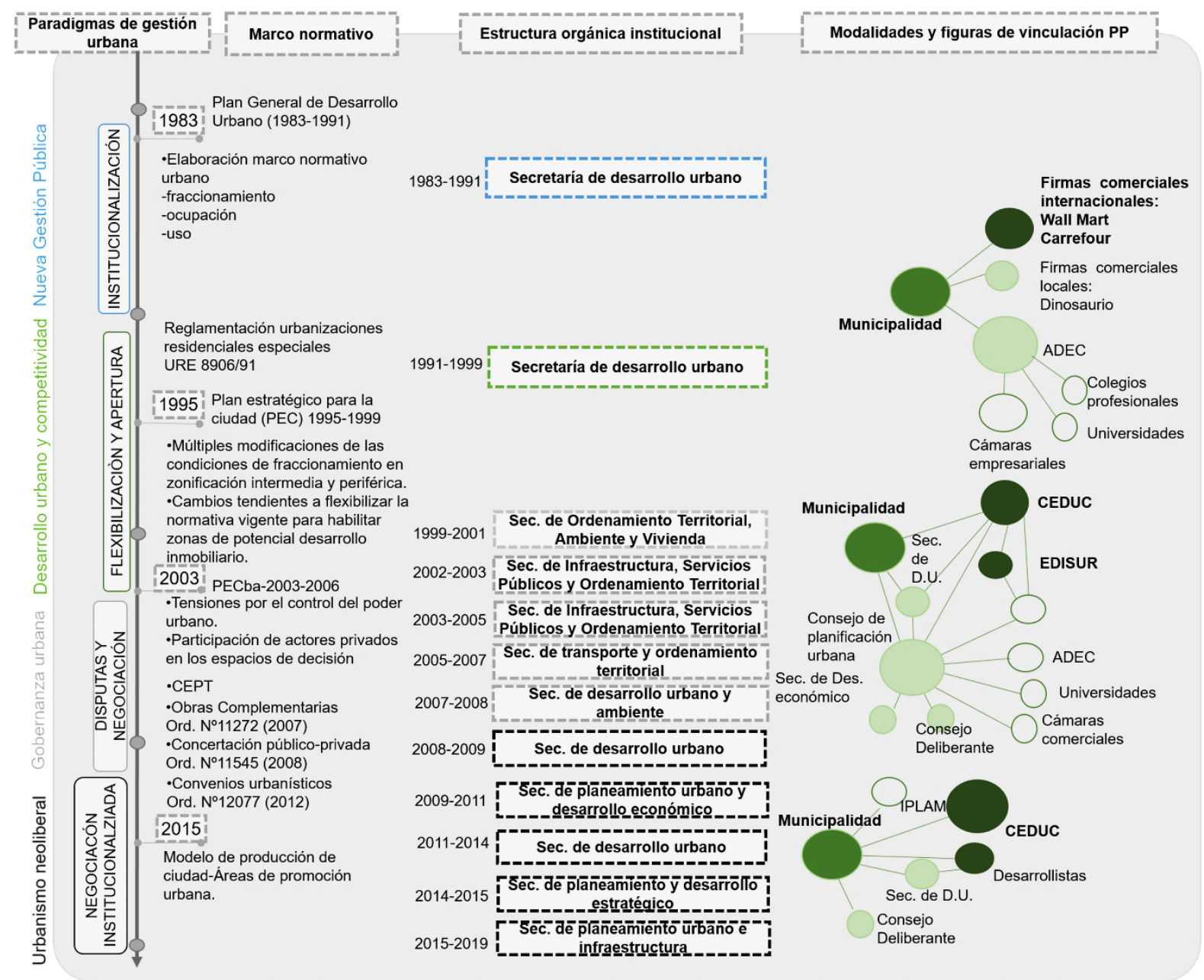

Figura 5 - Esquema síntesis de fases entre 1983-2019 según la caracterización de normativa urbana propuesta, estructura orgánica institucional y mecanismos y modalidades de vinculación público-privadas. Ciudad de Córdoba. Fuente:

Elaboración propia de acuerdo con documentos oficiales.

La genealogía nos permite exponer que en los últimos años se han consolidado los mecanismos dispuestos a mercantilizar y negociar las condiciones normativas del marco regulatorio urbano, en pos de asegurar un mejor escenario a las inversiones y operaciones urbano-inmobiliarias. Así también, la 
incorporación de instrumentos específicos, pensados y elaborados con la participación exclusiva de los actores a quienes va a afectar, marcan un ciclo novedoso de cambios institucionales y regulatorios. En este marco, y a lo largo de más de tres décadas, se han encadenado decisiones coadyuvadas por diferentes estrategias de presión por parte de los mismos desarrollistas inmobiliarios que han generado un diseño institucional y regulatorio favorable a sus demandas.

Podemos decir que lo realmente efectivo en este proceso ha sido la evolución de los mecanismos de vinculación entre el gobierno local y los empresarios desarrollistas. La revisión pudo evidenciar que desde los años noventa, el despliegue de mecanismos de vinculación público-privado ha sido constante, que aun siendo de distinto alcance y conformación siempre han podido ir un paso adelante en su legitimización e injerencia sobre las decisiones de la gestión urbana local. Todo ello, bajo un modelo de gestión urbana en el que se plantea la necesidad de un "cogobierno" en el que resulta necesario implementar innovadores mecanismos que permitan a los actores empresariales configurar, negociar y convenir las condiciones de regulación con el fin de mantener el desarrollo urbano.

La revisión y periodización de las adaptaciones normativas, regulatorias e institucionales expresan apenas una dimensión del impacto de la consolidación de la gobernanza empresarialista sobre la gestión urbana local. Partiendo de comprender que el gobierno local cuenta con la potestad jurídica para la regulación del suelo urbano, las sucesivas adaptaciones a las demandas del sector desarrollista se pueden interpretar entonces como una condición de la gobernanza. Paradigma que lejos de intentar garantizar la participación de diversos sectores, se ha consolidado como un modelo de gestión abocado a operacionalizar los intereses empresariales. Este proceso, desarrollado de forma proactiva aunque no siempre explícita, ha logrado insertar a los actores empresariales en los espacios de decisión de la gestión urbana local.

Igualmente importante en este proceso, resultan las formas institucionales que se desarrollan para darle soporte y legitimidad a estos vínculos. Como explica Araujo (2010), esta articulación es presentada ante la sociedad como una discusión racional entre los grupos afectados y los representantes de gobierno mediante instancias de participación que legitiman las decisiones. La secuencia de concertaciones, estrategias, acuerdos, asociaciones público-privadas se presentan como mecanismos diversos que expresan el mismo objetivo. En este sentido la cada vez mayor participación en las decisiones de gestión y políticas urbanas obtenida por los agentes ligados al complejo urbano-inmobiliario y la mayor institucionalidad conseguida por las distintas modalidades de vinculación y asociación, transparentan a los actores con acceso al poder urbano y demuestran la exitosa evolución de los arreglos guiados por el enfoque de la gobernanza urbana empresarialista.

\section{Bibliografía}

Araujo, A. E. (2010). Las nuevas formas de regulación neoliberal. Hendu - Revista Latino-Americana de Direitos Humanos, $1(1), 52-66$.

Baer, L. (2008). Crecimiento económico, mercado inmobiliario y ausencia de política de suelo. Un análisis de la expansión del espacio residencial de la Ciudad de Buenos Aires en los 2000. Revista Proyección, 2(5), 245.

Benavides, M., \& Gómez-Restrepo, C. (2005). Métodos en investigación cualitativa: triangulación. Revista Colombiana de Psiquiatria, 34(1), 118-124.

Brenner, N., Peck, J., \& Theodore, N. (2011). ¿Y después de la neoliberalización? Estrategias metodológicas para la investigación de las transformaciones regulatorias contemporáneas. Urban, 1(1), 21-40.

Brenner, N., \& Theodore, N. (2002). Cities and the Geographies of “Actually Existing Neoliberalism”. Antipode, 34(3).

Capdevielle, J. (2016). El mercado inmobiliario y la producción privada de viviendas: una aproximación a las estrategias empresariales en la ciudad de Córdoba (Argentina). Cuadernos de Geografía: Revista Colombiana de Geografía, 25(2), 177196. 
Cisterna, C., Monayar, V., \& Pedrazzani, C. (2012). Estructura urbana y estructura de precios del ciudad de CórdobaArgentina. Breves Contribuciones del I.E.G., 4176(23), 31-53.

Del Río, J., Vértiz, F., \& Ursino, S. (2014). La acción pública en el espacio urbano. Debates y reflexiones en torno a la noción de política urbana. Estudios Sociales Contemporáneos, 11, 76-86.

Díaz Orueta, F., \& Lourés, M. L. (2013). Neoliberalismo, políticas urbanas y reconfiguración socio-espacial. Quid 16, 3, 716.

Graglia, J. E. (2017). Políticas Públicas: 12 retos del siglo 21. Buenos Aires: Konrad Adenauer Stiftung editores.

Harvey, D. (1989). From managerialism to entrepreneurialism: the transformation in urban governance in late capitalism. Geografiska Annaler. Series B, Human Geography, 71(1), 3-17.

Harvey, D. (2007). Neoliberalism as Creative Destruction. The ANNALS of the American Academy of Political and Social Science, 610(1), 21-44.

Hidalgo, R., \& Janoschka, M. (eds.). (2014). La Ciudad Neoliberal. Gentrificación y exclusión en Santiago de Chile, Buenos Aires, Ciudad de México y Madrid. Santiago de Chile: Instituto de Geografía, Pontificia Universidad Católica de Chile y Departamento de Ciencia Política y Relaciones Internacionales, Universidad Autónoma de Madrid.

Iros, G. (1991). Desarrollo urbano. Reflexiones y acciones. Córdoba: Editorial municipal de la ciudad de Córdoba.

Jiménez, V., Campesino, A. J., Hidalgo, R., \& Alvarado, V. (2018). Normalización del modelo neoliberal de expansión residencial más allá del límite urbano en Chile y España. Eure, 44(132), 27-46.

Levenzon, F. (2012). La función social de la propiedad en términos de derechos humanos: implicaciones para la reforma del Código Civil. Revista Argentina de Teoría Jurídica, 12.

López, A. (2005). Los fundamentos de la Nueva Gestión Pública: lógica privada y poder tecnocrático en el Estado mínimo. In M. T. Rey, \& A. López, (comps.). Entre tecnócratas globalizados y políticos clientelistas. Derrotero del ajuste neoliberal en el Estado argentino (pp. 1-13). Buenos Aires: Prometeo.

López Morales, E., Gasic, I., \& Meza, D. (2012). Urbanismo pro-empresarial en Chile: políticas y planificación de la producción residencial en altura en el pericentro del Gran Santiago. Revista INVI, 27(76), 75-114.

Oszlak, O., \& O’Donnell, G. (1982). Estado y políticas estatales en América Latina: hacia una estrategia de investigación. REDES Revista del Centro de Estudios e Investigaciones de la Universidad Nacional de Quilmes, Buenos Aires, (4).

Ouviña, H. (2002). El Estado: su abordaje desde una perspectiva teórica e histórica. In S. Lifszyc (ed.). Introducción al conocimiento de la Sociedad y el Estado. Buenos Aires: Gran Aldea Editores.

Peck, J. \& Tickell, A. (2002). Neoliberalizing Space. Antipode, july 34. 380 - 404.

Peck, J., Theodore, N., \& Brenner, N. (2009). Neoliberal Urbanism: Models, Moments, Mutations. SAIS Review, 29(1), 4966.

Theodore, N., Peck, J., \& Brenner, N. (2009). Urbanismo neoliberal: la ciudad y el imperio de los mercados. Temas Sociales SUR, 66, 12.

Valles, M. (1999). Técnicas cualitativas de investigación social. En M. Valles. Reflexión Metodológica y Práctica Profesional (2a ed.). Madrid: Editorial Síntesis.

Van Lindert, P. (2016). Rethinking urban development in Latin America: A review of changing paradigms and policies. Habitat International, 54, 253-264.

Vicuña del Río, M. (2013). El marco regulatorio en el contexto de la gestión empresarialista y la mercantilización del desarrollo urbano del Gran Santiago, Chile. Revista INVI, 28(August 2013), 181-219.

\section{Fuentes}

Agencia para el desarrollo económico de la ciudad de Córdoba, ADEC (1997). ADEC. Primera Memoria anual.

Dávila, D. (2013, febrero 24). Tierra cada vez más cara y escasa. Diario La Voz Del Interior.

Digesto municipal de la ciudad de Córdoba. (2019). Ordenanzas y documentos oficiales. 
La consolidación de la gobernanza empresarialista en la gestión urbana local

Dirección de estadísticas general de la Provincia de Córdoba. (2017). Informe de producto geográfico bruto.

Guevara, V. (2008, marzo 9). Giacomino lanza su apuesta a la concertación. LaVoz.

Instituto Nacional de Estadísticas y Censos, Indec. (2010). Censo Nacional de Población, Hogares y Viviendas. Buenos Aires, Argentina.

LaVoz. (2004, junio 2). Empresarios quieren seguridad jurídica y mayor financiamiento. Diario La Voz Del Interior.

LaVoz. (2013, octubre 28). El valor de la tierra en cordoba. La Voz Del Interior.

Marianacci, G. (2000). Descentralización y desarrollo económico local: el estudio de caso de la ciudad de Córdoba, Argentina. Santiago: CEPAL.

Municipalidad de la Ciudad de Córdoba. (1996). Plan Estratégico para la Ciudad PEC. Córdoba: Editorial municipal de la ciudad de Córdoba.

Vanella, R., Lucca, C., Pittari, J. R., Steinberg, F., Zwanenburg, M., \& Cruz, S. (2001). El Plan Estratégico y el Desarrollo Económico Local de la Ciudad de Córdoba, Argentina.

Editor: Paulo Nascimento Neto

Recibido: 07 abr. 2020

Aprobado: 05 oct. 2020 